\title{
Fatores determinantes na taxa de homicídios da população do sexo masculino no Brasil: uma análise empírica com base em dados em painel, por unidades da federação no período 1993-2014
}

Determining factors in the homicide rate of the male population in Brazil: an empirical analysis based on panel data, by federation units in the period 1993-2014

Factores determinantes en la tasa de homicidios de la población masculina en Brasil: un análisis empírico basado en datos de panel, por unidades de la federación en el período 1993-2014

Recebido: 20/09/2021 | Revisado: 25/09/2021 | Aceito: 26/09/2021 | Publicado: 27/09/2021

Tito Belchior Silva Moreira

ORCID: https://orcid.org/0000-0002-2382-1480 Universidade Católica de Brasília, Brazil

E-mail: titoeco@yahoo.com.br

Lacerda Sipriano Elias

ORCID: https://orcid.org/0000-0002-4484-2950 Universidade Católica de Brasília, Brazil

E-mail: lacerdastones@gmail.com

\begin{abstract}
Resumo
Este artigo avalia, empiricamente, os fatores determinantes das taxas de homicídios na população do sexo masculino no Brasil. O estudo aborda o assunto com dados em painel com efeito fixo e efeito aleatório por Unidades da Federação no período 1993-2014. Discute-se, utilizando outras variáveis explicativas, o impacto do desemprego nas taxas de homicídios dessa população. Com base nos dois modelos em painel, efeito fixo e efeito aleatório, os resultados atestam a hipótese de que aumentos na taxa de desemprego produzem elevações nas taxas de homicídios da população masculina. As variáveis, grau de informalidade, percentual da renda adquirida pelas pessoas pertencentes ao grupo dos $50 \%$ mais pobres e a despesa com educação e cultura também são estatisticamente significantes no estudo. De um modo geral, as variáveis trabalhadas confirmaram os resultados esperados.
\end{abstract}

Palavras-chave: Homicídio; Desemprego; Dados em painel.

\begin{abstract}
This article empirically assesses the determinants of homicide rates in the male population in Brazil. The study approaches the subject with panel data with fixed effect and random effect by Federation Units in the period 19932014. Using other explanatory variables, the impact of unemployment on the homicide rates of this population is discussed. Based on the two panel models, fixed effect and random effect, the results support the hypothesis that increases in the unemployment rate produce increases in homicide rates in the male population. The variables, degree of informality, percentage of income earned by people belonging to the poorest $50 \%$ group and expenditure on education and culture are also statistically significant in the study. In general, the worked variables confirmed the expected results.
\end{abstract}

Keywords: Homicide; Unemployment; Panel data.

\section{Resumen}

Este artículo evalúa empíricamente los determinantes de las tasas de homicidio en la población masculina en Brasil. El estudio aborda el tema con datos de panel con efecto fijo y efecto aleatorio por Unidades de la Federación en el período 1993-2014. Utilizando otras variables explicativas, se discute el impacto del desempleo en las tasas de homicidio de esta población. Con base en los dos modelos de panel, efecto fijo y efecto aleatorio, los resultados apoyan la hipótesis de que los aumentos en la tasa de desempleo producen aumentos en las tasas de homicidio en la población masculina. Las variables, grado de informalidad, porcentaje de ingresos obtenidos por personas pertenecientes al grupo más pobre del $50 \%$ y gasto en educación y cultura también son estadísticamente significativas en el estudio. En general, las variables trabajadas confirmaron los resultados esperados.

Palabras clave: Homicidio; Desempleo; Datos de panel. 


\section{Introdução}

De acordo com Atlas da Violência (2021), 57.956 homicídios foram registrados no Brasil no ano de 2018 e desse total de pessoas vitimadas, aproximadamente, 92,2\% eram pessoas do sexo masculino. Embora, em 2019, seja observada uma redução aproximada de 21,49\% no número total de homicídios e, aproximadamente, 21,84\% no número de homicídios de homens, a quantidade de pessoas que perderam suas vidas é alarmante.

A criminalidade é um tema de debate diário, pois deixa em alerta toda a sociedade. De acordo com Paula (2010), os mais diversos cenários sociais presenciam a violência. Silva (2007) argumenta que inclusive as edificações urbanas brasileiras têm sofrido alterações em função da violência.

Waiselfisz (2014) sugere que desde as últimas décadas do século XX tem ocorrido uma reestruturação na modelagem do desenvolvimento produtivo no Brasil e esse fator tem contribuído para o aumento da criminalidade.

Silva (2011) aponta os elevados índices de homicídios observados no Brasil, o que torna essencial o fomento de pesquisas de caráter preventivo, ou seja, que impeçam a concretização da ação criminosa. De fato, é necessário um conjunto de pesquisas interdisciplinares que permitam a implementação de políticas públicas integradas que reduzam a violência no Brasil.

Essa pesquisa discute os determinantes da taxa de homicídio na população do sexo masculino, no período 1993-2014, utilizando dados em painel, com efeito fixo e efeito aleatório, por Unidades da Federação. As variáveis explicativas utilizadas nessa pesquisa são o PIB per capita, índice de Theil, proporção da renda adquirida pelas pessoas pertencentes ao conjunto dos 50\% mais pobres de acordo com a distribuição da renda domiciliar per capita, a despesa com educação e cultura, o grau de informalidade e a variável de interesse, isto é, a taxa de desemprego, que fundamenta nossa hipótese, pois acreditamos evidenciar uma relação direta com a taxa de homicídios da população masculina brasileira.

O trabalho está distribuído em cinco seções. Além dessa introdução, a revisão da literatura é apresentada na seção 2, a metodologia, que inclui base de dados e modelos econométricos, está na seção 3, resultados e discussão na seção 4 e, na seção 5 são apresentadas as considerações finais.

\section{Revisão da Literatura}

De acordo com Atlas da Violência (2021) a taxa de homicídios por 100 mil habitantes, no Brasil, que era 27,2 em 2009, registrou 21,7 em 2019, isto é, uma redução de aproximadamente 20,22\% e embora as taxas de homicídios de vítimas do sexo masculino também apresentem diminuição no período, esses percentuais ainda se mostram muito elevados.

A criminalidade produz transtornos e gastos adicionais para a sociedade. Os homicídios destroem os sonhos das famílias vitimadas e o registro de taxas tão elevadas refletem um número alarmante de vidas e talentos que deixam de contribuir para o desenvolvimento pessoal, familiar e do país.

Estudos interdisciplinares são importantes para a implementação de políticas públicas integradas que reduzam essas taxas e proporcionem segurança, paz e tranquilidade para a sociedade.

Nesse sentido, é essencial o estudo e planejamento de ações preventivas. Para Cano e Ribeiro (2007), ações que caracterizam os crimes quanto ao perfil das vítimas e o diagnóstico da tipologia e localidade do fato ocorrido se complementam para a prevenção de homicídios. Adicionalmente, as coletas de dados e as mensurações dos índices são essenciais para analisar, interpretar e buscar as melhores soluções para a redução das taxas de criminalidade no país. Oliveira e Souza (2019) comentam a importância da taxa de homicídios para analisar o comportamento eleitoral, pois o aumento da violência agrava o bem estar geral da sociedade e, consequentemente, pode produzir impactos em eleições. 
Spaniol, Júnior e Rodrigues (2020) em análise bibliográfica e documental das Políticas Públicas de Segurança já adotadas, argumentam que para constatar a ocorrência de reduções e ações preventivas eficazes na criminalidade há necessidade de participação social na elaboração dessas políticas que precisam se manter contínuas.

Nogueira, Gomes e Barbosa (2018), em estudo sobre homicídios em Foz do Iguaçu e Estado do Paraná no período 2010-2015, apontam para uma redução das taxas dessa modalidade de crime no período estudado, contudo destacam que a faixa etária mais vitimada é composta por adultos jovens do sexo masculino, dessa forma, constata-se um fato preocupante, pois são integrantes de uma grande parcela da população produtiva do país.

Silva (2019) estuda determinantes de homicídios nos municípios do Estado da Paraíba e observa que no período 2005 2015, aproximadamente, 56\% do total dos homicídios registrados são de jovens na faixa etária de 15 a 29 anos de idade e desses, $93 \%$ são do sexo masculino.

Souza, Souza e Pinto (2020), estudando homicídios no Estado da Bahia no período 2013-2015, destacam uma relação inversa entre o nível de educação e as taxas de homicídio e, para as localidades estudadas, fica evidenciado que as ações que melhoram os índices educacionais funcionam como um fator protetivo para as cidades. Moraes e Chaves (2020) argumentam, também, a necessidade de investimentos em educação e profissionalização que gerem renda e autonomia financeira para os adolescentes em conflito com a lei.

Souza Lucas, Cunha e Bondezan (2020), ao analisar aspectos socioeconômicos determinantes da criminalidade no Estado do Paraná, mostram que o elevado grau de desigualdade econômica e altas taxas de desemprego indicam tendências para taxas de homicídios mais elevadas.

Mendonça, Loureiro e Sachsida (2003) apontam a existência de uma relação direta entre desigualdade social e as taxas de criminalidade e Fajnzylber, Lederman e Loayza (2002) argumentam que elevações na desigualdade de renda produzem aumentos na taxa de criminalidade.

Olini, Daniel, Dalfovo, Orlandi e Shikida (2018), em estudo com análise espacial para o Estado do Mato Grosso no período 2000-2010, observam uma relação direta entre desigualdade de renda e taxa de homicídios nas localidades estudadas.

Ervilha e Lima (2019), em dados em painel com efeito fixo para estudar os determinantes da criminalidade em municípios de Minas Gerais no Brasil, argumentam que as lacunas observadas no mercado de trabalho podem estar agravando a criminalidade no Estado mineiro.

Regateiro, Ramos, Souza e Mello (2021), avaliando criminalidade no Estado do Pará no período 2017-2019, constatam que a maioria dos municípios paraenses apresentou redução nos índices de criminalidade, contudo aqueles que se encontram destacados no ranking com os mais elevados índices, evidenciaram reduzidas taxas de ocupação para o trabalho e, também, péssimas condições de saneamento básico e urbanização.

Pinto, Farias, Costa e Lima (2018), em painel dinâmico, analisam os determinantes das taxas dos crimes de homicídios nas Unidades da Federação do Brasil e encontram uma relação direta da variável renda média e taxa de homicídios e ao confrontar a taxa dessa modalidade de crime com o grau de informalidade, observa uma relação inversa, contudo não significante no estudo. No entanto, Oliveira (2019) estuda os impactos da criminalidade em Fortaleza - Ceará e observa uma relação inversa entre renda média e taxas de homicídio nos bairros estudados.

Becker e Kassouf (2017) argumentam que reduções nas taxas de crime podem ser observadas em função dos aumentos nos gastos públicos em educação, considerado um período para percepção do efeito, enquanto as estimativas do estudo de Gomes (2019) sugerem uma redução de, aproximadamente, 0,4\% no total de homicídios no Brasil, caso ocorra um aumento de $1 \%$ nas despesas com segurança. 
Em modelos estudados por Loureiro, Moreira e Ellery (2017), a desigualdade de renda e a taxa de pobreza são variáveis significantes para explicar a taxa de homicídio e observam que aumentos na desigualdade conduzem a elevações na taxa de homicídios, enquanto aumentos na taxa de pobreza, redução.

Máquina, Maria, Nhogo e Come (2021), em estudo sobre criminalidade nos municípios do Estado de São Paulo, verificam que a combinação de pobreza extrema e inacessibilidade à educação, que contribui para elevar as taxas de analfabetismo nas localidades estudadas, têm sido fatores importantes e determinantes para aumentos da violência urbana e, consequentemente, do número de homicídio.

\section{Metodologia}

Kauark, Manhães e Medeiros (2010) argumentam que a análise quantificável que utiliza recursos e técnicas estatísticas, dentre elas a análise de regressão, caracteriza a pesquisa quantitativa. Essa pesquisa quantitativa utiliza o método indutivo para abordar o assunto. De acordo com Marconi e Lakatos (2017), esse método é mais abrangente na abordagem dos fenômenos, pois procura chegar a leis e teorias gerais partindo de constatações particulares. O objetivo da pesquisa é descritivo e segundo Gil (2008), a descrição da característica de um fenômeno ou a determinação das relações entre as variáveis estudadas representa o objetivo principal desse tipo de pesquisa.

Essa seção está dividida em duas subseções. A subseção 3.1 apresenta as fontes e as definições dos dados utilizados na pesquisa e a seção 3.2 apresenta o modelo econométrico considerado.

\subsection{Base de dados}

Nesse trabalho, estamos interessados em analisar os determinantes das taxas de homicídio na população do sexo masculino, utilizando uma análise com dados em painel com efeito fixo e efeito aleatório, considerando o período 1993-2014 para todas as unidades da federação do Brasil, relacionadas no modelo econométrico apresentado na subseção 3.2. O Quadro 1, a seguir, relaciona as variáveis utilizadas, os dados oficiais e as fontes de coleta, o período e o sinal esperado nos resultados.

Quadro 1 - Fontes e definições dos dados adotados.

\begin{tabular}{|c|c|c|c|c|}
\hline Variável & Período & Fonte & & Sinal esperado \\
\hline $\begin{array}{l}\text { Taxa de homicídios } \\
\text { do sexo masculino } \\
\text { (homich) }\end{array}$ & $1993-2014$ & Descrição & $\begin{array}{l}\text { "Óbito por causa externa ou não natural, indiferente } \\
\text { do tempo entre o evento lesivo e a morte } \\
\text { propriamente, é categorizado como consequente de } \\
\text { lesão provocada por violência (acidentes, homicídios, } \\
\text { suicídios ou morte suspeita). Neste caso, a taxa por } \\
100.000 \text { habitantes é calculada através da divisão do } \\
\text { indicador principal (número de homicídios do sexo } \\
\text { masculino) pelo total da população em questão, sendo } \\
\text { este resultado multiplicado por 100.000. Fonte: Os } \\
\text { dados originais são provenientes do SIM-DATASUS. } \\
\text { Até } 1995 \text { as informações são do CID9. A partir de } \\
1996 \text { são do CID10. Elaboração Ipeadata: Cálculo da } \\
\text { Taxa, divisão do grupo populacional multiplicado por } \\
100.000 \text { pela população de referência". }\end{array}$ & \\
\hline $\begin{array}{c}\text { Índice de Theil. } \\
\text { (theil) }\end{array}$ & 1993-2014 & IPEADATA/ IPEA & $\begin{array}{l}\text { "Mede a desigualdade na distribuição de indivíduos } \\
\text { segundo a renda domiciliar per capita. Série calculada } \\
\text { a partir das respostas à Pesquisa Nacional por } \\
\text { Amostra de Domicílios (Pnad/IBGE)". }\end{array}$ & Positivo \\
\hline $\begin{array}{l}\text { Despesas por } \\
\text { função - educação e } \\
\text { cultura - Estadual. } \\
\quad(\text { despec }) \text {. }\end{array}$ & 1993-2014 & $\begin{array}{l}\text { IPEADATA/Minist } \\
\text { ério da Fazenda/ } \\
\text { Secretaria r do } \\
\text { Tesouro Nacional. }\end{array}$ & $\begin{array}{l}\text { "Estão discriminadas as despesas segundo rubrica: } \\
\text { Educação e Cultura". } \\
\text { Unidade: } \mathrm{R} \$ \text { (por bilhão). }\end{array}$ & Negativo \\
\hline
\end{tabular}




\begin{tabular}{|c|c|c|c|c|}
\hline $\begin{array}{l}\text { Taxa de } \\
\text { desemprego } \\
(\text { desemp })\end{array}$ & 1993-2014 & IPEADATA /IBGE & $\begin{array}{l}\text { "Percentual das pessoas que procuraram, mas não } \\
\text { encontraram ocupação profissional remunerada entre } \\
\text { todas aquelas consideradas "ativas" no mercado de } \\
\text { trabalho, grupo que inclui todas as pessoas com } 10 \\
\text { anos ou mais de idade que estavam procurando } \\
\text { ocupação ou trabalhando na semana de referência da } \\
\text { Pesquisa Nacional por Amostra de Domicílios } \\
\text { (Pnad)". }\end{array}$ & Positivo \\
\hline $\begin{array}{c}\text { Grau de } \\
\text { informalidade. } \\
\text { (inform) }\end{array}$ & 1993-2014 & IPEADATA /IBGE & $\begin{array}{l}\text { Grau de informalidade - definição I } \\
\text { "Uma das três diferentes definições do grau de } \\
\text { informalidade oferecidas no IPEADATA com base na } \\
\text { Pesquisa Nacional por Amostra de Domicílios (Pnad) } \\
\text { do IBGE, esta taxa corresponde ao resultado da } \\
\text { seguinte divisão: (empregados sem carteira + } \\
\text { trabalhadores por conta própria) / (trabalhadores } \\
\text { protegidos + empregados sem carteira + trabalhadores } \\
\text { por conta própria). Elaboração: Disoc/IPEA". } \\
\text { Unidade: }(\%)\end{array}$ & Positivo \\
\hline $\begin{array}{l}\text { PIB estadual per } \\
\text { capita. } \\
(p i b p c) \\
\end{array}$ & 1993-2014 & IPEADATA/ IBGE & $\begin{array}{l}\text { "PIB Estadual a preços constantes (série calculada } \\
\text { pelo Ipeadata), dividido pela população". }\end{array}$ & Negativo \\
\hline $\begin{array}{c}\text { Renda domiciliar - } \\
\text { participação dos } \\
50 \% \text { mais pobres. } \\
(\text { renpob })\end{array}$ & 1993-2014 & IPEADATA/ IPEA & $\begin{array}{l}\text { "Proporção da renda apropriada pelos indivíduos } \\
\text { pertencentes ao grupo dos } 50 \% \text { mais pobres da } \\
\text { distribuição, segundo a renda domiciliar per capita. } \\
\text { Série calculada a partir das respostas à Pesquisa } \\
\text { Nacional por Amostra de Domicílios (Pnad/IBGE)". } \\
\text { Unidade: }(\%)\end{array}$ & Negativo \\
\hline
\end{tabular}

Fonte: Autores.

O método de construção aborda dados em painel com efeito aleatório e efeito fixo. Em Gujarati (2019), os modelos destinados a estudar as entidades que pertencem ao mesmo grupo, ao longo do tempo, são chamados de modelos de regressão de dados em painel. Segundo o autor, ao presumir que para cada indivíduo, o coeficiente específico individual B1i seja fixo no tempo, tem-se o efeito fixo e, efeito aleatório caso esse coeficiente seja uma variável randomizada com valor médio de B1.

Hausman (1978) apresenta um teste para verificar qual dos dois modelos, efeito fixo ou efeito aleatório é o mais apropriando e, de acordo com o autor, ao aceitar a hipótese nula o efeito aleatório é o mais indicado e, em caso de rejeição, o efeito fixo.

\subsection{Modelo econométrico}

Nesse estudo, o modelo econométrico estimado relaciona a taxa de homicídio de indivíduos do sexo masculino (variável dependente) com as variáveis explicativas, no qual a principal variável de interesse é a taxa de desemprego, procurando verificar se, possivelmente, essa impacta nas taxas de homicídio dessa população. A equação de homicídios do conjunto em estudo está definida a partir de dados em painel de acordo com a seguinte forma:

$H_{\text {it }}=\beta x_{i t}+\gamma_{t}+v_{i t}$ para $i=1_{v \ldots,} 27, t=1_{v, 2} 22$

em que $H_{\text {it }}$ é a taxa de homicídio de indivíduos do sexo masculino da $i$-ésima Unidade da Federação no período t. A matriz contém intercepto, $x_{i t}$ representa o vetor de variáveis explicativas, $v_{i t}$ é o termo aleatório e $\gamma_{t}$ visa captar efeitos específicos no tempo. Segundo a metodologia para dados em painel, define-se ainda que $v_{i t}=\alpha_{i}+u_{i t}$, no qual $\alpha$ é um termo estocástico próprio das unidades. Generalizando: 
$H_{\text {it }}=\beta x_{i t}+\gamma_{t}+\alpha_{i}+u_{i t}$ para $i=1, \ldots, 27, t=1, \ldots 22$

Assim, $i$ representa a $i$-ésima unidade de corte transversal e $t$ o $t$-ésimo período de tempo. Se cada unidade de corte transversal estiver presente o mesmo número de observações de séries temporais, então esse painel recebe a denominação de painel equilibrado.

Segundo Wooldridge (2010), a abordagem clássica de dados em painel trata de verificar se o componente individual ( $\propto)$ é ou não correlacionado com algum regressor. No primeiro caso, o modelo deve ser estimado por meio da aplicação de um estimador denominado de efeito fixo. No segundo caso, o mais apropriado é estimar o modelo por efeito aleatório. Para se verificar qual das duas hipóteses é a que melhor se aplica, emprega-se o teste de Hausman.

Segundo Wooldridge (2010) a estimação de $H_{\text {it }}$ depende das premissas que são realizadas a respeito do intercepto, dos coeficientes angulares e do termo de erros, $u_{\text {it }}$.

Nesse estudo estamos considerando o modelo:

$H_{\text {it }}=f\left(x_{1}, x_{2}, x_{3}, x_{4}, x_{5}, x_{6}\right)$

$H_{\text {it }}=$ taxa de homicídio de indivíduos do sexo masculino.

$x_{l}=$ indice de Theil .

$x_{2}=$ grau de informalidade.

$x_{3}=$ pib per capita

$x_{4}=$ taxa de desemprego.

$x_{5}=$ Proporção da renda apropriada pelos indivíduos pertencentes ao grupo dos $50 \%$ mais pobres.

$x_{6}=$ despesa em educação e cultura.

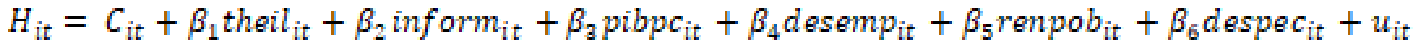

com $i=1,2,3, \ldots, 27$ et $=1993,1994,1995, \ldots, 2014$.

Os dados são utilizados para a construção de um estudo econométrico em dois modelos em dados em painel, efeito fixo e efeito aleatório, considerando a taxa de homicídio de indivíduos do sexo masculino como a variável dependente e o restante das variáveis registradas no Quadro 1, como variáveis explicativas. No modelo 1 a abordagem é realizada em efeito aleatório e no modelo 2 , em efeito fixo.

\section{Resultados e Discussão}

Os resultados empíricos são apresentados em 2 diferentes modelos econométricos, que tem como foco a variável explicativa de interesse nessa pesquisa, ou seja, a taxa de desemprego.

As estimativas apresentadas e enunciadas a seguir, com efeitos aleatórios e fixos, têm como base uma análise em dados em painel no período 1993 a 2014, considerando-se todas as unidades da federação do Brasil. Os resultados empíricos são revelados na Tabela 1 seguinte, com os respectivos comentários. 
Tabela 1 - Determinantes da taxa de homicídio de indivíduos do sexo masculino no Brasil. Dados em painel, efeito aleatório e efeito fixo, período 1993-2014.

\begin{tabular}{|c|c|c|c|c|}
\hline Variável & \multicolumn{2}{|c|}{ Modelo 1 - Efeito aleatório } & \multicolumn{2}{|c|}{ Modelo 2 - Efeito fixo } \\
\hline & Coeficiente & $P>|t|$ & Coeficiente & $P>|t|$ \\
\hline Theil & $\begin{array}{l}-13,317 \\
(13,446)\end{array}$ & 0,322 & $\begin{array}{r}-14,375 \\
(13,630)\end{array}$ & 0,292 \\
\hline Inform & $\begin{array}{l}-0,813 * * * \\
(0,194)\end{array}$ & 0,000 & $\begin{array}{l}-0,845 \text { *** } \\
(0,204)\end{array}$ & 0,000 \\
\hline Pibpc & $\begin{array}{l}-0,054 \\
(0,393)\end{array}$ & 0,891 & $\begin{array}{l}-0,129 \\
(0,544)\end{array}$ & 0,811 \\
\hline Desemp & $\begin{array}{l}1,520 * * * \\
(0,341)\end{array}$ & 0,000 & $\begin{array}{l}1,354 * * * \\
(0,353)\end{array}$ & 0,000 \\
\hline Renpob & $\begin{array}{l}2,146 * * \\
(0,857) \\
\end{array}$ & 0,012 & $\begin{array}{l}2,241 * * \\
(0,920) \\
\end{array}$ & 0,015 \\
\hline Despec & $\begin{array}{l}-1,906 * * * \\
(0,325)\end{array}$ & 0,000 & $\begin{array}{l}-1,985 \text { *** } \\
(0,334)\end{array}$ & 0,000 \\
\hline $\mathrm{C}$ & $\begin{array}{l}65,944 * * \\
(28,855) \\
\end{array}$ & 0,022 & $\begin{array}{l}69,679 * * \\
(29,417)\end{array}$ & 0,018 \\
\hline
\end{tabular}

Entre parênteses estão os erros padrões. Nota: $(* * *)$ significante a $1 \%$; $(* *)$ significante a $5 \%$; $(*)$ significante a $10 \%$. Modelo $1: 513$ observações, $R^{2}=0,1463$. Modelo 2: 513 observações, Prob $>F=0,0000, R^{2}=0,2682$. Fonte: Autores.

i) O teste de Hausman não aceita a hipótese nula com uma estatística do Qui-quadrado no valor de 21,36 com 6 graus de liberdade e uma probabilidade menor que 0,01. Nesse contexto, o efeito fixo é o mais indicado.

ii) A taxa de desemprego é estatisticamente significativa nesse estudo e o sinal positivo do coeficiente mostra que aumentos nessa variável produzem elevações nas taxas de homicídios da população masculina no Brasil.

iii) Ambos, índice de Theil e a variável PIB per capita, não são estatisticamente significativos nesse estudo. Contudo, o PIB per capita apresenta o sinal negativo esperado, sugerindo que aumentos na produção de bens e serviços, com os respectivos aumentos per capita, podem favorecer a oferta de vagas no mercado de trabalho formal, reduzindo o desemprego e assim, produzir impactos na diminuição dos crimes de homicídios na população masculina brasileira.

iv) A variável, grau de informalidade, é estatisticamente significante nesse estudo e apresenta uma relação inversa com a variável dependente, mostrando que aumentos na informalidade produzem efeitos redutores nas taxas de homicídios da população brasileira. Dessa forma, os resultados podem sugerir que a ocupação laboral, mesmo que informal, tem característica redutora da criminalidade.

v) Os resultados obtidos com a variável despesa em educação e cultura correspondem às expectativas esperada. Mostra a relação inversa existente entre essa variável explicativa e a dependente, ou seja, aumentos nos gastos educacionais e culturais impactam significativamente, conforme os resultados evidenciados, nas reduções das taxas de homicídios da população estudada.

vi) A variável, percentual da renda apropriada pelas pessoas pertencentes ao grupo dos $50 \%$ mais pobres, é estatisticamente significante nesse estudo e o sinal positivo do coeficiente mostra a relação direta com a variável dependente, isto é, mostra que quanto maior o percentual dessa renda, maiores serão as taxas de homicídio da população em questão. Embora o sinal não seja o esperado, o resultado sugere ações de segurança adicionais e complementares nas áreas habitadas pela população proprietária dessas rendas.

Por fim, a variável explicativa de interesse, isto é, a taxa de desemprego, confirma a hipótese assumida nessa pesquisa. Por conseguinte, constata-se a relação direta dessa variável explicativa com a variável dependente, ou seja, a taxa de homicídios da população masculina no Brasil. Os resultados sugerem estudos e ações adicionais, complementares 
e interdisciplinares para a implementação de políticas públicas que produzam impactos diretos na geração de empregos formais.

\section{Considerações Finais}

Homicídios, além de produzirem angústias e tristezas para as famílias vitimadas, geram perdas para o setor produtivo e para o mercado de trabalho, pois as vidas perdidas para a violência reduzem a quantidade de talentos da demanda laboral.

Essa pesquisa utiliza dados sobre taxas de homicídios da população masculina no Brasil e variáveis socioeconômicas para testar e confirmar a hipótese de que aumentos na taxa de desemprego impactam positivamente nas taxas dessa modalidade de crime, no grupo citado.

O estudo aborda em dois modelos com dados em painel por efeito fixo e aleatório, no período 1993-2014 por Unidades da Federação do Brasil, os determinantes das taxas de homicídio da população masculina brasileira, utilizando a taxa de desemprego, o PIB per capita, o índice de Theil, o grau de informalidade, o percentual da renda adquirida pelas pessoas pertencentes ao grupo dos 50\% mais pobres e a despesa com educação e cultura como variáveis explicativas.

Considerando a variável dependente taxa de homicídios do grupo estudado e as variáveis explicativas citadas, o teste de Hausman indicou que dentre os dois modelos construídos, o efeito fixo mostrou-se o mais apropriado, com os resultados apresentados na seção 4.

As variáveis, índice de Theil e PIB per capita, não são estatisticamente significantes nesse estudo, embora o sinal negativo observado para o PIB per capita mostra uma relação inversa com a variável dependente, sinalizando que aumentos na produção de bens e serviços podem conduzir a um aumento na oferta de empregos e, por conseguinte impactar na redução das taxas de homicídios da população masculina.

A variável despesa em educação e cultura é estatisticamente significativa e apresenta o resultado esperado, ou seja, mostra que aumentos em investimentos nessas áreas produzem impactos na redução dos crimes de homicídio no grupo estudado. Adicionalmente, sugere políticas públicas voltadas para a promoção e divulgação de eventos educacionais e culturais que alcancem a população.

A variável, grau de informalidade, é estatisticamente significante e a relação inversa com a variável dependente sugere que a ocupação laboral, mesmo que informal, produz impactos redutores nas taxas de homicídios do grupo estudado nesse trabalho.

O coeficiente positivo obtido no estudo da variável, percentual da renda adquirida pelas pessoas pertencentes ao conjunto dos 50\% mais pobres, indica uma relação direta com a variável taxa de homicídios. Embora estatisticamente significativa, o sinal obtido não era esperado, contudo sugere planejamentos de segurança adicionais e complementares nas áreas habitadas pela população proprietárias dessas rendas.

Para concluir, a variável de interesse nessa pesquisa, a taxa de desemprego, confirma a hipótese assumida. Assim, constata-se uma relação direta com a variável taxa de homicídios da população masculina brasileira e o resultado sugere o estudo de políticas públicas para a geração de empregos na modalidade formal, pois a redução do desemprego se apresenta como importante redutor das taxas de homicídios da população estudada.

Esse trabalho não esgota o assunto, no entanto contribui para a literatura ao propor um estudo que aborda os impactos das taxas de desemprego sobre as taxas de homicídio da população do sexo masculino, que ao longo dos anos vem registrando índices elevados no número de vítimas nessa modalidade de crime.

Destaca-se como possibilidade de trabalhos futuros, a formulação de novos modelos com outras variáveis explicativas e suas combinações, que possam contribuir para ampliar e enriquecer a literatura sobre o assunto aqui estudado. 
Research, Society and Development, v. 10, n. 12, e474101220808, 2021 (CC BY 4.0) | ISSN 2525-3409 | DOI: http://dx.doi.org/10.33448/rsd-v10i12.20808

\section{Referências}

Becker, K. L. \& Kassouf, A. L. (2017). Uma Análise do Efeito dos Gastos Públicos em Educação Sobre a Criminalidade no Brasil. Economia e Sociedade. $26(1), 215-42$.

Cano, I. \& Ribeiro, E. (2007). Homicídios no Rio de Janeiro e no Brasil: dados, políticas públicas e perspectivas. In: Cruz MUG, Batitucci ECO, eds. Homicídios no Brasil. FGV, 51-78.

Cerqueira, D. (2021). Atlas da violência 2021. IPEA.

Ervilha, G. T., \& Lima, J. E. D. (2019). Um método econométrico na identificação dos determinantes da criminalidade municipal: a aplicação em Minas Gerais, Brasil (2000-2014). Economía, sociedad y territorio, 19(59), 1059-1086.

Fajnzylber, P., Lederman, D., \& Loayza, N. (2002). What causes violent crime? European economic review, 46(7), $1323-1357$.

Gil, A. C. (2008). Métodos e técnicas de pesquisa social. (6a ed.), Ediitora Atlas SA.

Gomes, C. (2019). Criminalidade e despesas públicas no Brasil: estimativa do impacto dos gastos públicos em segurança sobre as taxas de homicídio (No. IDB-WP-909). IDB Working Paper Series.

Gujarati, D. (2019). Econometria: Princípios, teoria e aplicações práticas. Saraiva Educação SA.

Hausman, J. A. (1978). Specification tests in econometrics. Econometrica: Journal of the econometric society, 1251-1271.

Kauark, F. D. S., Manhães, F. C., \& Medeiros, C. H. (2010). Metodologia da pesquisa: um guia prático.

Loureiro, P. R., Moreira, T. B. S., \& Ellery, R. (2017). The relationship between political parties and tolerance to criminality: A theoretical model and empirical evidences for Brazil. International Journal of Social Economics.

Máquina, C. M., Maria, E. da C. J., Nhongo, E. J. S., \& Come, S. F. (2021). Desigualdade social como vetor dos homicídios no Estado de São Paulo, Brasil. Research, Society and Development, 10(12), e176101220237.

Marconi, M. D. A., \& Lakatos, E. M. (2017). Fundamentos de metodologia científica. (8a ed.), Atlas.

Mendonca, M. J. C., Loureiro, P. R., \& Sachsida, A. (2003). Criminality and Social Interaction.

Moraes, M. J. S. M., \& Chaves, A. B. P. (2020). Adolescente infrator: reincidência e vitimização por homicídio. Research, Society and Development, 9(8), e496985998-e496985998.

Nogueira, V. D., Gomes, L. M. X., \& de Andrade Barbosa, T. L. (2018). Tendência da mortalidade por homicídio em Foz do Iguaçu e Paraná, 2010 a 2015. Revista Brasileira de Iniciação Científica, 5(2), 222-234.

Olini, R. M., Daniel, L. P., Dalfovo, W. C. T., Orlandi, M., \& Shikida, P. F. A. (2018). Homicídio e desigualdade de renda: uma análise espacial para o estado de Mato Grosso em 2000 e 2010. Economic Analysis of Law Review, 9(3), 107-130.

Oliveira, A., \& Souza, F. B. (2019). A taxa de homicídio explica a escolha do eleitor? Revista Observatório, 5(1), $478-496$.

Oliveira, D. X. A. D. (2019). Os impactos da criminalidade em uma análise teórica, empírica e espacial.

Paula, J. C. D. (2010). Aqui tem violência? as representações sociais de violência urbana dos moradores da Ceilândia.

Pinto, A. M., Farias, J. J. Costa, R. F., \& Lima, F. S. (2018). Uma análise dos determinantes da taxa de crimes de homicídios nos estados do Brasil: uma aplicação em painel dinâmico. Revista de Economia Regional, Urbana e do Trabalho, 7(2), 35-52.

Regateiro, H. A. S., Ramos, E. M. L. S., Souza, J. G., \& Mello, C. M. de A. (2021). Avaliação da criminalidade no Estado do Pará. Research, Society and Development, 10(3), e10010313088.

Silva, F. M. D. (2019). Homicídio de jovens e sua conexão com a pobreza e a desigualdade nos Municípios Paraibanos.

Silva, J. (2011). A prevenção da violência e criminalidade no Brasil: causas, fatores, experiências de sucesso e alternativas. UFSC.

Silva, R. O. (2007). Violência e juventude: um estudo de representações sociais em Uberlândia-MG.

Sousa Lucas, M., da Cunha, M. S., \& de Lucas Bondezan, K. (2020). Determinantes socioeconômicos da criminalidade no estado do Paraná: uma análise espacial. Revista de Economia, 41(75).

Souza, T. O. D., Souza, E. R. D., \& Pinto, L. W. (2020). Análise da correlação entre fatores socioeconômicos, sanitários, demográficos e óbitos por homicídioBahia, Brasil, 2013-2015. Revista Brasileira de Enfermagem, 73.

Spaniol, M. I., Moraes Jr, M. C., \& Rodrigues, C. R. G. (2020). Como tem sido planejada a Segurança Pública no Brasil? Análise dos Planos e Programas Nacionais de Segurança implantados pós-redemocratização. Revista Brasileira de Segurança Pública, 14(2), $100-127$.

Waiselfisz, J. J. (2014). Os jovens do Brasil: mapa da violência 2014. Secretaria Nacional de Juventude.

Wooldridge, J. M. (2010). Introdução à econometria: uma abordagem moderna. Cengage Learning. 